\title{
Urinary NGAL levels in potential deceased kidney donors may be useful in determining donor suitability
}

Urinary levels of neutrophil gelatinaseassociated lipocalin (NGAL) may be useful in determining the suitability of a potential deceased kidney donor, according to a Finnish study.

Delayed graft function (DGF) is a common complication in recipients of kidneys from deceased donors, and-if prolonged-has a negative effect on graft survival. "As the number of deceased donors has remained approximately constant during the last decades, but the need for kidney transplants has increased, we have been forced to relax the donor criteria, which means that older donors with more comorbidities are accepted for transplantation," states Maria Hollmén, an author on the study. "Although the etiology of DGF is multifactorial, we have found that the rate of DGF has grown as an increased number of older and marginal donors have been accepted for transplantation. If we could assess the kidney quality of a potential donor already in the intensive care unit it would really help in deciding whether or not the kidneys are suitable for transplantation."

NGAL is a promising marker for acute kidney injury and is useful in a number of clinical settings. As little is known about whether NGAL levels could be useful in kidney transplantation, however, Hollmén et al. set out to determine whether donor NGAL levels can be used to predict DGF.

\section{Although the etiology of DGF is multifactorial, we have found that the rate of DGF has grown... 77}

The study included 99 consecutive, deceased, heart-beating donors and the 176 adult recipients who received kidneys from these donors. Donor clinical history data were obtained from hospital records and recipient data obtained from hospital records and the Finnish Kidney Transplant Registry database.
DGF occurred in $39.8 \%$ of transplanted kidneys (mean onset of graft function was 12 days after transplantation). Kidneys from donors with urine NGAL levels higher than the mean $(\geq 18 \mathrm{ng} / \mathrm{ml})$ were more likely to show prolonged DGF (DGF lasting > 14 days) and had worse 1-year graft survival; however, receiver operating curve analysis showed that the predictive power of urine NGAL levels for DGF was poor. Serum NGAL levels were not associated with post-transplantation DGF.

"We have started to measure donor NGAL levels routinely to gather more data on how donor NGAL levels associate with a recipient's kidney function after transplantation," says Hollmén.

\section{Rebecca Ireland}

Original article Hollmen, M. E. et al. Deceased donor neutrophil gelatinase-associated lipocalin and delayed graft function after kidney transplantation: a prospective study. Crit. Care doi:10.1186/cc10220 